\title{
DE LA RESPONSABILITÉ ÉNONCIATIVE À LA RESPONSABILITÉ JURIDIQUE EN DROIT DE LA PRESSE BELGE*
}

\author{
Emmanuël Murhula-Amisi Nashi' ${ }^{1}$
}

Un certain imaginaire collectif largement ancré dans nos sociétés considère généralement le journaliste comme la figure centrale de la production médiatique. Ceci n'est pas sans fondement, car le travail journalistique demeure l'essence même de la presse ${ }^{2}$. Dans le même ordre d'idées, la conception qui consiste à placer le journaliste au centre de la plupart des législations sur la presse nous semble inspirée par cet imaginaire-là.

Depuis des décennies, le droit de la presse belge, en particulier, a assuré une immunité à des acteurs qui portent des responsabilités, et non des moindres, sur l'énonciation de la presse : éditeur, imprimeur,

* Nous remercions Jean-Pierre Meunier, Benoît Grevisse et Jacques Polet pour leurs précieuses remarques.

1 Docteur en sciences sociales (information et communication), Département de communication de l'Université catholique de Louvain.

2 Nous consacrons à ce terme une acception restreinte, c'est-à-dire la presse écrite, quotidienne ou hebdomadaire d'informations générales. Il va de soi qu'une définition plus large inclura les nouveaux moyens de communication (radio, télévision, informatique...). Voir la mise au point sur la définition de la presse faite par C. Doutrelepont et D. Fesler, in G. Thoveron et C. Doutrelepont (dir.), La presse, pouvoir en devenir, Bruxelles, Revue de l'Université Libre de Bruxelles, 1996, pp. 194-195.

Recherches en communication, $\mathrm{n}^{\circ} 17$ (2002). 
distributeur, rédacteur en chef, secrétaire de rédaction. En somme, jusqu'à une récente décision judiciaire condamnant solidairement un éditeur et son journaliste ${ }^{1}$, on avait systématiquement fait endosser à un seul la responsabilité judiciaire d'une énonciation à l'élaboration de laquelle beaucoup participent.

En soi, la décision du tribunal civil de Bruxelles constitue une révolution. Et ce n'est pas seulement parce que ce jugement a sonné le glas de l'immunité systématisée des éditeurs. C'est aussi et surtout parce que pour la première fois, un juge reconnaissait l'existence, dans un article signé, de parties non attribuables à son auteur². À notre sens, cet arrêt constitue une des manifestations de ce que nous qualifierions, à la suite de Jacques Polet, de "changement de culture jurisprudentielle"3 observé depuis quelques années en Belgique. Aussi, il s'inscrit dans un mouvement dynamique où se meut la réflexion juridique sur la responsabilité des acteurs de la presse.

Le présent article se propose de jeter sur le champ disciplinaire du droit un regard venu d'ailleurs, en l'occurrence des sciences de la communication. Il s'agit d'approcher la notion de "responsabilité juridique" en rapport avec un sujet revêtu d'un statut particulier. Ce sujet qui agit par la parole à travers la presse écrite. Les articles de presse eux-mêmes sont à considérer comme des messages communicationnels, et ce, dans une perspective interdisciplinaire. ainsi :

La communication comme interdiscipline, nous l'entendons

Étude des actes de communication socialement élaborés, des significations portées par ces actes, des relations et

1 L'éditeur responsable de La Dernière Heure-Les Sports, Patrick le Hodey, et le journaliste Gilbert Dupont avaient été assignés par une infirmière de la Clinique Latour de Uccle, à la suite d'un article publié par le journal sous le titre "Bernadette, la deuxième pyromane". Le quotidien fut condamné pour violation de la présomption d'innocence, violation de la vie privée, et publication d'une photo de l'infirmière légendée "la vamp mini-jupée".

2 La décision rendue le 25 avril 2000 par la $14^{\circ}$ chambre du tribunal civil de Bruxelles rendait l'éditeur responsable des parties de l'article non attribuables à un journaliste déterminé : la photo, la légende, les encarts, les titres et sous-titres. Cf. M. Simonis, "Bernadette n'était pas pyromane", Journalistes. La lettre de $l^{\prime} A J P, \mathrm{n}^{\circ} 12$, janvier 2001, p. 3.

3 C'est-à-dire une mutation opérée dans la culture judiciaire et rompant "spectaculairement avec la tradition". Cela concernait, chez l'auteur, la condamnation des journalistes d'investigation au paiement de montants élevés au titre de dommages et intérêts. Voir J. POLET, "Les procès civils de presse: un changement de culture ?", La Revue Nouvelle, septembre 2001, pp. 58-65. 
phénomènes cognitifs sollicités par ceux-ci, des représentations socialement partagées qui y sont liées et des effets des représentations sur les rapports sociaux ${ }^{1}$.

Dans cet entendement, cette réflexion tente un dialogue entre le droit de la presse et la communication, sous l'éclairage de notions comme l'énonciation, la pragmatique, la polyphonie, le contexte, etc.

Il n'est donc pas question ici d'un questionnement juridique. Il s'agit d'un apport contextuel au raisonnement consistant à soutenir l'évolution de la jurisprudence vers un partage des responsabilités en matière de presse. En d'autres termes, nous entendons argumenter d'un point de vue communicationnel dans le même sens que les juristes belges qui jugent anachronique le fait que le journaliste continue à payer seul pour les dommages causés à des tiers par ses articles, et qui plaident, de ce fait, pour une reconfiguration du champ de la responsabilité.

\section{Responsabilité et énonciation}

La responsabilité trouve ses fondements dans sa filiation au terme "imputer", qui signifie : "attribuer l'action à quelqu'un comme à son véritable auteur". Au plan juridique, l'auteur est responsable des effets de ses actes, "que ceux-ci soient des pensées, des paroles ou des actions"2.

Quant aux contours de la notion d'auteur, ils se posent en des termes qui en révèlent toute la complexité, surtout lorsque cela concerne la presse :

qui parle dans les pages d'information d'un grand quotidien ? Est-ce la dépêche d'agence, le journaliste qui la développe et qui signe éventuellement l'article, celui qui lui met un chapeau ou un titre, le rédacteur en chef (...), est-ce la "ligne" du journal qui inspire chaque article, et cette ligne monte-t-elle des lecteurs ou descend-elle des propriétaires du journal ou de ses plus gros annonceurs ? $?^{3}$.

1 "Recherches en communication", Recherches en communication, n' 1, 1994, p. 1.

2 P. RICEUR, "Le concept de responsabilité. Essai d'analyse sémantique", Esprit, $n^{\circ} 206,1994$, pp. 30 et 38.

3 D. Bougnoux, La communication contre l'information, Paris, Hachette, 1995, pp. 97-98. 
Mouillaud et Tétu abordent la question du sujet de la presse d'un point de vue analogue au nôtre, c'est-à-dire celui de la communication. Ils observent, notamment, à quel point la question (qui parle ?) est beaucoup plus générale que la simple signature d'un journaliste en bas d'un article. Pour cette raison, écrivent-ils ${ }^{1}$ :

Il faut tenir compte de l'organisation, de la propriété, de la gestion des différents médias ; des "pesanteurs" socio-professionnelles des journalistes, des rapports entre les journalistes situés à des stades différents du circuit de l'information; et surtout des rapports de ces professionnels avec ceux qui sont souvent les sources de leur information, mais qui ont des buts non journalistiques en fournissant celle-ci².

Par ailleurs, si l'on en croit Charaudeau, il faut craindre que :

S'agissant des médias, on ne sait jamais vraiment qui peut répondre d'une information, même lorsqu'elle s'accompagne de la signature d'un journaliste, tant les effets de l'instance médiatique de production transforment les intentions de la seule instance d'énonciation discursive... ${ }^{3}$.

De ce point de vue, une jonction est indispensable, concernant la question du sujet et de sa responsabilité, entre le droit et les théories du langage. Ce lien peut s'établir, entre autres, par le truchement des théories pragmatiques. D'abord, parce que ces dernières pensent un sujet qui, par sa parole, agit (dire, c'est faire). Ensuite, parce qu'ici l'accent est mis, non plus sur l'énoncé (son sens et sa référence) mais sur l'énonciation.

Or, l'énonciation c'est principalement un procès. Procès dans lequel l'acte de langage, n'est plus appréhendé à son état d'achèvement, mais dans un processus global qui advient dans un contexte précis : un temps, un espace, et des sujets. L'énonciation, dans cette conception, concerne l'acte d'auto-désignation par lequel le sujet se

1 M. Mouillaud et J.-F. TETU, Le journal quotidien, Lyon, Presses Universitaires de Lyon, 1989, pp. 37-38.

2 Ibid., p. 40.

3 P. ChaRAUdeaU, Le discours d'information médiatique. La construction du miroir social, Paris, Nathan, 1997, p. 82. 
désigne lui-même comme tel, en laissant des traces de sa présence sur son acte'.

Comment définir, dans cet ordre d'idées, l'énonciation de la presse ? De notre point de vue, l'article de presse est à appréhender comme une énonciation imprimée. Il faut savoir, en effet, que l'écrit qu'un journaliste remet à sa rédaction est un manuscrit ${ }^{2}$, et celui-ci ne devient un article qu'après avoir été programmé, relu et corrigé, mis en pages, et enfin imprimé et publié dans la presse. Tel est bien le sens de l'énonciation de la presse.

C'est dans ce sens qu'il faut comprendre, à la suite de Bronckart, la responsabilité énonciative dont la question est à formuler comme suit: "quelles sont les instances qui prennent en charge ce qui est énoncé dans le texte ? Quelles sont les voix qui s'y expriment ?"3.

Lamarque l'exprime sans doute mieux : il y a, d'après lui, une "inévitable responsabilité" du journal(iste) qui se trouve engagée, au minimum, du seul fait de l'énonciation publique d'une information ${ }^{4}$. Là devrait se situer aussi, à notre sens, la source de la responsabilité de la presse. Il convient donc d'examiner le contexte par lequel cette énonciation vient au public.

\section{Le contexte d'énonciation de la presse}

Considérant le processus de production, il est établi que la matière journalistique est présentée de manière agréable à l'aide des possibilités techniques (impression en couleur, lay-out aéré, infographie) et des moyens pratiques rendant le texte plus agréable au lecteur (encadrés, repères, renvois, mots clés, etc.). Et il ne s'agit pas là d'un

1 On trouvera un développement plus complet de cette notion chez C. KERBRATORECCHIONI, L'énonciation. De la subjectivité dans le langage, $3^{\circ}$ éd., Paris, Armand-Colin, 1980, pp. 29-30.

2 En jargon journalistique, le manuscrit désigne tout texte remis à la rédaction avant une éventuelle publication. Qu'il soit écrit à la main, tapé à la machine, ou saisi sur ordinateur.

3 J.-P. BRONCKART, Activité langagière, textes et discours. Pour un interactionisme socio-discursif, Neuchâtel-Paris, 1996, p. 130. Roger Odin aussi envisage la responsabilité énonciative en termes d'authentification. Le responsable du discours sera donc pour lui, "celui qui garantit l'authenticité des événements racontés et des paroles prononcées" (cité par M. HaNOT, La télévision, réalité ou réalisme, Bruxelles, De Boeck \& Larcier, 2002, p. 18).

${ }^{4}$ P. LAmarque, "La presse écrite face à ses devoirs", Médiaspouvoirs, n 31-32, 1993, pp. 158-159. 
simple emballage. Des "énonciateurs dans l'ombre"' interviennent, et ce, de manière parfois déterminante, sur le discours. Rappelons également que la décision de juger publiable ou non, de garder silence (black-out) sur un événement, constitue un choix croisé du journaliste et de son rédacteur en chef. De ce point de vue, la responsabilité énonciative est partagée.

En outre, il est admis aujourd'hui que l'activité journalistique se trouve soumise à des contraintes d'ordre économique, à des mécanismes de contrôle idéologique, et à des modèles quasi imposés d'écriture $^{2}$ qui réduisent considérablement les marges d'initiative individuelle.

On pourrait ajouter à cela les phénomènes de concentrationsfusions caractérisant le contexte socio-économique actuel, y compris dans les entreprises de presse ${ }^{3}$, et qui rognent progressivement l'indépendance effective des journalistes, conséquence inéluctable de la précarité ${ }^{4}$ qui élit domicile au sein de la profession.

On vit, en Belgique aujourd'hui, dans un contexte de chômage :

La course aux suppressions "douces" d'emplois va bon train, des signatures de collaborateurs extérieurs disparaissent, le nombre de pages passe au régime-minceur et bien des

1 A savoir : secrétaire de rédaction, rédacteur en chef, lay-out, monteur, metteur en page, etc. Voir E. MuRHULA AMISI NASHI, Le méga-énonciateur, sujet du discours de la presse. Approche sémio-pragmatique de la polyphonie scripto-visuelle, thèse de doctorat, inédite, Département de communication, U.C.L., 2001-2002.

2 Voici un témoignage: "Je me demandais toujours pourquoi, à l'occasion de l'intervention d'un ministre, tous les journaux, les radios et les télés retenaient tous, sans exception, la même phrase. J'ai vite compris : les trois correspondants des médias les plus importants (AFP, Le Monde et TF 1) se réunissaient dans un coin et déterminaient, ensemble, ce qui deviendrait la phrase clé du discours" (P. COHEN, "Qui sont les journalistes ?", Marianne, 23-29 avril 2001, p. 60).

3 Ce qui a suscité une certaine inquiétude de la part de nombreux organismes et instances internationaux. Voir F. DUBUISSON, "Le contrôle de concentration de la presse et le maintien du pluralisme dans les droits français, belge et européen", in G. THOVERON et C.DOUTRELEPONT (dir.), op. cit., pp. 191-213. Sur la concentration de la presse quotidienne en Belgique, voir E. LENTZEN, "Concentration et presse quotidienne", in G. THOVERoN et C. DOUTRELEPONT, op. cit., pp. 227-255.

4 La précarité, montante dans la profession (20\% des journalistes sont précaires), facilite les choses, c'est-à-dire la "souplesse" de ces journalistes", dixit P. COHEN (art. cit., p. 63). Pour quelques exemples des dérives qu'entraîne la précarité (publiinformation, corruption passive, petits et gros cadeaux, etc.), voir le chapitre IX de J.-M. Charon., Cartes de Presse. Enquête sur les journalistes, Paris, Stock, 1993, pp. 289-298. On pourra lire également les témoignages de journalistes, comme A. ACCARDo et al., Journalistes précaires, Bordeaux, Le Mascaret, 1998. 
hypothèses de nouvelles restrictions budgétaires sont imaginées"1

Dans ces conditions, l'indépendance du journaliste risque de se voir relativisée, par rapport au risque que représenterait la perte de son emploi.

Dès lors, la qualité d'employé du journaliste ${ }^{2}$ devrait être établie, et sa responsabilité revisitée par rapport à celle de son employeur quant aux articles rédigés dans le cadre de l'exercice de ses fonctions. Faut-il le rappeler, juridiquement, la relation entre le journaliste et sa publication est dominée par le "lien de subordination".

D'aucuns, tels Lochard et Boyer ${ }^{3}$, en sont venus à conclure que toute production médiatique est mise en œuvre par des collectifs professionnels, et non par des sujets individuels. Sans pouvoir les suivre jusque dans la négation de l'individu, on retiendra néanmoins que la presse adopte généralement un point de vue forgé à partir d'opinions multiples : celles des propriétaires, des plus gros annonceurs, des membres de la rédaction et de la fabrication, du lectorat, et de la déontologie. Vu sous cet angle, derrière les apparences d'une centration sur l'énonciateur individuel, le discours de la presse fonctionnerait sur des mécanismes de centration - décentration 4 .

Mais, il s'agit souvent d'une décentration feinte, car en réalité on a affaire à la multiplication d'un même point de vue. Les personnalités, les individus, les groupes auxquels on ouvre ses colonnes, y compris dans le choix qui est fait du courrier des lecteurs, sont généralement des voix qui renforcent la position du journal. Est-on fondé à affirmer que le droit pour chaque journal à une position

1 J.-F. Dumont, "Presse : avis de tempête", Le Vif/L'Express, 19e année, n 44, 2-8 novembre 2001, p. 18.

2 On aura compris, nous l'espérons, que nous ne parlons pas du "free-lance", journaliste indépendant, qui n'est pas employé par une entreprise particulière, mais vend ses articles aux organes de presse de son choix.

3 G. LOCHARD et H. BOYER, La communication médiatique, Paris, Éd. du Seuil, 1998, p. 13.

4 Ces notions sont analysées par J.-P. MEUNIER et D. PERAYA, dans Introduction aux théories de la communication, Bruxelles, De Boeck Université, 1993, pp. 204-208. Elles désignent respectivement la façon dont une unité sociale (individu, groupe) s'éprouve comme centre du monde, autosuffisant, replié sur soi et marginalisant ce qui l'entoure. A l'inverse la décentration est ce phénomène par lequel un être social manifeste une certaine capacité de dépassement de soi, d'ouverture vers l'autre, de prise en compte de la position de ce dernier, de compréhension de son point de vue, de sa pensée, de son vécu, etc. 
idéologique (rédactionnelle) empêcherait la presse de jouer son rôle de pourvoyeur d'opinions multiples?

Dans tous les cas, on touche ici à une des raisons pour lesquelles les journaux d'un même groupe de presse offrent souvent le même contenu rédactionnel, en termes d'opinion tout au moins. En Belgique, E. Lentzen en est persuadée :

Que vous habitiez Virton et achetiez L'Avenir du Luxembourg, que vous travailliez à Dinant et êtes abonné à Vers l'Avenir, que vous cherchiez Le Jour/Le Courrier à Verviers, Le Rappel à Charleroi ou Le Courrier de l'Escaut à Tournai, vous lisez le même journal. À quelques pages près, écrit-elle'1.

II faut cependant observer que la presse n'est pas seule responsable d'une telle situation, car les lecteurs jouent avec elle un jeu de gratification réciproque. Boutot, dont les travaux portent un intérêt sur la responsabilité du public dans le comportement de la presse, pense que "la responsabilité morale de l'objectivité s'est déplacée du journaliste vers le lecteur. C'est le lecteur, désormais, qui choisit ses propres sources d'information, les confronte et en tire sa propre interprétation". Dans un contexte où l'éducation est généralisée, les libertés de l'information, de la circulation et de la concurrence des médias garantie, renchérit-il, c'est le lecteur qui cautionne le credo journalistique en achetant tel ou tel journal ${ }^{2}$.

Or, le comportement moyen du lecteur dans les démocraties occidentales face à la presse pourrait se résumer en une sorte de "prophétie auto-réalisatrice". En général, les gens lisent un journal (qu'ils achètent ou auquel ils sont abonnés) dont ils attendent qu'il conforte leurs propres convictions, ce que le titre leur rend par ailleurs.

En effet, d'une part, jouissant d'une position énonciative privilégiée grâce à sa fonction sociale, la presse est censée rapporter l'opinion, c'est-à-dire ce que pense la société. D'autre part, et paradoxalement, ce sont des individus, en l'occurrence les journalistes, qui parlent pour les gens en leur (re) donnant un point de vue présenté comme étant celui de la société. De rapporteuse d'opinions, la presse

I E. Lentzen, "Concentration et presse quotidienne", in G. Thoveron et C. DOUTRELEPONT, op. cit, pp. 227-255.

2 B. BouTot, "Le débat information/interprétation. «Connais ton lecteur»", in J.M. Charon (dir.), L'état des médias, Paris, La Découverte-Médiaspouvoirs-CFPJ, 1991, pp. 38-39. 
se retrouve ainsi faiseuse d'opinion, transformant, sans toujours en prendre conscience, une opinion singulière, la sienne, en une opinion sociale, en l'opinion.

\section{Énonciation polyphonique, sujet pluriel}

On comprend que dans ces conditions Régis Debray ait écrit : "c'est toujours un individu qui émet ou reçoit tel message, mais l'énonciation est généralement polyphonique et plurielle, surtout quand elle se médiatise"1.

Selon notre point de vue aussi, le discours de la presse est, effectivement, envisageable comme une polyphonie, c'est-à-dire comme une énonciation composée de plusieurs énonciations. Cette notion, entrée en littérature par M. Bakhtine, a été reprise et enrichie par des auteurs tels que Ducrot, Récanati, Adam, Bronckart et beaucoup d'autres pour caractériser une phrase, un texte littéraire ou bien tout autre énoncé monologique qui intègre le discours d'autrui ou une "voix" autre que celle de l'énonciateur de l'intervention".

La problématique polyphonique, rappelle pour sa part Maingueneau ${ }^{3}$, touche à la question de l'identité du sujet énonciateur. Elle s'est développée dans le sillage d'auteurs pour qui, il faut distinguer, pour rendre compte d'un certain nombre de phénomènes linguistiques, entre trois statuts différents qu'assume, le plus souvent, celui qui profère un énoncé. Entre le "sujet parlant" (l'individu qui physiquement dit ou écrit l'énoncé), le "locuteur" (la personne qui mobilise à son profit le système de la langue et se place à l'origine des repérages référentiels) et l'"énonciateur" (le responsable des actes illocutoires).

L'énonciation de la presse se compose, quant à elle, de trois voix $^{4}$ (matières sémiologiques) ou plutôt de trois voix en une, qui

1 Cité par D. BougnouX, Sciences de l'information et de la communication, Paris, Larousse, 1993, pp. 136-137.

2 C. Rubattel, "Polyphonie et modularité", Cahiers de linguistique française, $\mathrm{n}^{\circ} 11$, 1990, p. 297.

3 Voir D. Maingueneau, Éléments de linguistique pour le texte littéraire, Paris, Bordas, 1990, p. 69.

4 D'autres auteurs tels que G. Genette, A. Gardies, F. Jost, A. Gaudreault, mais surtout J.-F. Bronckart conçoivent également la "voix" dans une acception non auditive: "voix filmique" ou "voix sociale". 
nous conduisent à qualifier le discours de la presse de "polyphonie scripto-visuelle"1.

En la décomposant, on y distingue :

- une voix scripto, composée des éléments de l'article relevant du linguistique : titre, surtitre, sous-titre, chapeau, article, légende, nom de l'auteur, nom du journal ;

- une voix iconique, renfermant les éléments de l'illustration, c'est-à-dire les images, schémas, graphiques, dessins, etc. ;

- une voix graphique qui englobe le "typographique"(variations de forme, hauteur, largeur et épaisseur, orientation et couleur de la lettre) d'une part, et le "topographique" : utilisation de l'espace, mise en page, agencement des différentes matières différentes d'expression avec les autres éléments de la page, d'autre part.

Dans ce cadre, on parle de l'écriture de presse comme d'une "écriture scripto-visuelle". Une écriture qui, comme le souligne Henri Boyer, "peut jouer non seulement sur la dimension langagière, mais également sur les composantes iconiques, chromatiques, typographiques du spectacle journalistique"2. Car, ici plus qu'ailleurs, l'exercice du langage se révèle comme une mise en œuvre simultanée de plusieurs systèmes signifiants complémentaires les uns des autres ${ }^{3}$.

En réalité, il s'agit de bien plus qu'une complémentarité. En matière de presse, c'est véritablement de "contamination réciproque" qu'il faut s'occuper : entre texte et image ; entre analogique et digital ; entre écrit, photo et typographie ${ }^{4}$.

Il sied de souligner toutefois que c'est uniquement pour les besoins de l'analyse qu'il est possible de décomposer l'énonciation de la presse, car on n'a pas affaire à de l'écrit, plus de l'image, plus de la mise en pages. Il s'agit véritablement d'une énonciation nouvelle réunissant en elle seule les trois autres, et que l'on peut baptiser une "méga-énonciation"s.

1 E. MURHULA-Amisi NASHI, op. cit, pp. 186-187.

2 H. BOYER , "Scription et écriture dans la communication journalistique", in H. BOYER \& al., La presse. Produit, production, réception, Paris, Didier Érudition, 1988, p. 74.

3 A. BERRENDONNER, Les référents nominaux du français et la structure de l'énoncé, Lille, Atelier national de reproduction des thèses, 1983, p. 20.

$4 \mathrm{Ph}$. MARION, "Propositions pour une médiatique narrative appliquée", in J. BAETENS \& A. GoNZALEZ, Le roman photo, Amsterdam, Rodopi, 1996, p. 163.

5 E. Murhula-Amisi NASHI, op. cit, pp. 306-308. On notera que le discours filmique aussi est considéré par A. Gaudreault (Du littéraire au filmique. Système du récit, Paris, Méridiens Klincksieck, 1989, p. 115) comme un "méga-récit". 
Schématiquement, le dispositif de méga-énonciation de la presse se structure comme suit :
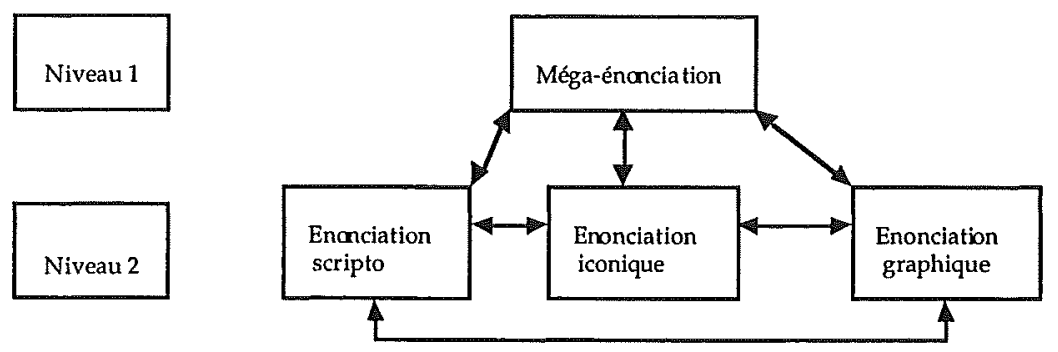

Comme on peut le voir, les trois énonciations du niveau 2 sont prises en charge par une instance particulière, c'est-à-dire un énonciateur. Et puis, au premier niveau se situe une instance générale d'énonciation qui régit ou distribue les voix, ces dernières désignant les "voix secondes" (voix de personnages, voix sociales, voix de l'auteur). C'est cette instance supra qui assume directement la responsabilité du dire, ainsi que le démontrait Bronckart ${ }^{1}$.

Dans cette perspective, certains théoriciens des médias traitent du sujet d'énonciation de la presse comme d'une instance responsable de l'intégralité du message, les parties revenant, par délégation, à des sous-instances.

Chez Baylon et Mignot, par exemple, on peut voir se multiplier les sujets à qui le locuteur ${ }^{2}$ fait appel. Cependant, précisent ces auteurs, parmi eux, ne mérite pleinement d'être appelé énonciateur que celui qui parle pour son propre compte, "celui à qui revient la responsabilité intégrale du message"3.

Souchard aussi considère non seulement le journaliste, mais aussi le journal comme des énonciateurs. Il s'agit cependant d'énonciateurs à statuts différents, car le premier reçoit délégation du second. Pour cet auteur, en effet, rares sont les textes où le média prend directement la parole, plus rares encore sont ceux où le journaliste -et même l'éditorialiste-comme énonciateur délégué du média intervient dans l'énoncé 4 .

1 J.-P. BRONCKART, op. cit., pp. 324-325.

2 Dans leur terminologie, "le scripteur", en ce qui concerne la presse.

3 C. BAYlon \& X. Mignot, La communication, Paris, Nathan, 1991, pp. 91-93.

4 M. Souchard, Le discours de presse. L'image des syndicats au Québec, Québec, Préambule, 1989, p. 137. 
Pareilles décompositions analytiques pourraient accréditer la thèse de l'existence d'une hiérarchie, l'énonciateur délégué devant assumer moins de responsabilités que l'énonciateur lui-même. Au contraire, nous voudrions montrer que, au plan pragmatico-énonciatif, ces instances agissent de manière solidaire.

Les "énonciateurs" du deuxième niveau prennent en charge une partie seulement du discours, certes. Mais, si l'on veut bien considérer comme nous que les rapports prévalant entre les niveaux 1 et 2 sont de type systémique ${ }^{1}$, c'est-à-dire que cette énonciation est une unité complexe où le tout est plus que la somme des parties, on comprendra que toute intervention sur l'une des sous-énonciations produit une méga-énonciation nouvelle. Dans ces conditions, les énonciateurs ne sont plus isolés, au contraire, ils participent tous à la mégaénonciation, et la responsabilité devient coresponsabilité.

Voici trois exemples :

1. Au plan scripto : une virgule mal placée peut être mortelle... ${ }^{2}$

Dans un ouvrage médical, l'auteur indiquait un médicament à base de chlorure de sodium à $2,5 \%$ mais, lors de l'impression, la virgule avait été omise. Un médecin voulant expérimenter l'ordonnance sur un malade, lui administra la solution à $25 \%$, (selon l'indication de l'ouvrage) dans le temps prescrit. Le malade a pu être sauvé de justesse 3 .

2. Au plan iconique: un cadrage, une légende peuvent tout changer dans une illustration ${ }^{4} .$.

Guéry prévenait à ce sujet :

Rien n'est plus facile, en effet, que de déformer complètement le sens d'une photographie en la cadrant d'une certaine façon, par exemple en supprimant un ou plusieurs personnages

1 Du "système" tel que l'entend Edgar Morin, c'est-à-dire une "unité globale organisée d'interrelations entre éléments, actions ou individus" (E. Morin, cité par J.-P. MEUNIER, Théorie systémique de la communication (cours), Département de communication, U.C.L., Louvain-la-Neuve, 1998-1999, p. 20).

2 L. GUÉRY, Le secrétariat de rédaction : de la copie à la maquette de mise en page, Paris, C.F.P.J., 1990, p. 258.

3 Il faut avoir à l'esprit, en lisant ce passage, que sur le plan juridique, "la faute du journaliste peut être retenue pour des questions liées à la mise en forme ou à la mise en évidence de l'information : emploi de guillemets, du conditionnel, de la forme interrogative; emploi de titres, de dessins, de photos, d'images, de légendes...", (S. HOEBEKE \& B. MOUFFE, Le droit de la presse, Louvain-la-Neuve, Bruylant-Academia, 2000, p. 574).

4 Exemple tiré de L. GUÉRY, op. cit., p. 170. 
importants, en coupant un détail qui expliquait ou justifiait une attitude, en attirant l'attention sur une scène ou un objet qui n'était que secondaire, etc."1.

$3 \mathrm{Au}$ plan graphique : une coupure insidieuse peut se révéler source de confusions ${ }^{2} \ldots$

... DES COUPURES HASARDEUSES...

\section{EN ROUMANIE}

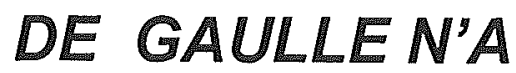

FAIT QU'UNE FOIS

allusion à la crise

universitaire

Au plan énonciatif, c'est clair à présent, les instances qui gèrent chacune de ces énonciations en portent la responsabilité. Non seulement celle-ci est engagée par rapport à chaque "sous-énonciation" elle-même, mais elle l'est aussi par rapport à la méga-énonciation.

Quant à l'énonciateur qui assume la méga-énonciation, c'est cette instance théorique fondamentale qui s'exprime au sein du discours de la presse, et que nous nommons, ailleurs, le "mégaénonciateur"' . Celui-ci intervient dans l'énoncé à titre d'instance donnant un "point de vue", une "position", qui ne s'expriment pas à travers des mots précis".

Il s'agit véritablement d'une instance sans nom, sans nom de personne, parce qu'elle n'est pas une personne mais précisément une instance, une instance de mise en pages, de mise en place, de mise en ordre, ce qui l'empêcherait et ce, de manière irréductible de dire "je"s.

1 Un photoreporter écrivait: "Nous savons qu'on peut, en modifiant un cadrage, changer ou déplacer l'accent mis sur tel ou tel comportement et provoquer chez le lecteur des réactions ou des interprétations totalement différentes" (Paul Almasy, cité par L. GUÉRY, op. cit., p. 171).

2 Exemple tiré de L. GUÉRY, op. cit., p. 122.

3 E. MurhUla-Amisi NASHI, op. cit, pp. 315-317.

4 D. Maingueneau, Éléments de linguistique pour le texte littéraire, Paris, Bordas, 1990, p. 77.

5 Ces traits descriptifs sont fondamentalement analogues à ceux du méga-narrateur de Gaudreault. Voir A. Gaudreault, cité parJ. Châteauvert, Des mots à 


\section{Responsabilité juridique individuelle}

Cependant, jusqu'à présent, le régime juridique fixant les responsabilités en matière de presse en Belgique semble s'être comporté d'une manière quelque peu simplificatrice face à ces considérations. Fondamentalement parce que, comme le signale Martine Simonis, les décisions judiciaires ont "très peu nuancé ce principe de droit, qui veut que l'auteur connu est seul responsable, même s'il s'imposait de constater que dans les faits, cette responsabilité était partagée avec d'autres acteurs"'. Ainsi, la nature énonciative des actes journalistiques, leur caractère polyphonique, et les effets systémiques caractérisant les comportements individuels des auteurs ont été peu présents dans les jugements et "attendus".

Le droit reconnaît pourtant une particularité à la presse, car le journaliste fait partie de la seule profession à bénéficier de deux dispositions spéciales : la clause de conscience et la clause de cession $^{2}$. Leur particularité réside en ce qu'elles permettent aux membres d'une rédaction qui apportent la preuve qu'il est advenu dans leur journal changement et situation nouvelle de nature à porter atteinte à leur honneur, à leur réputation ou, d'une manière générale, à leurs intérêts moraux, de quitter celui-ci en bénéficiant des indemnités égales à celles d'un licenciement.

Ils invoqueront la clause de conscience en cas de changement d'orientation idéologique notable, de volte-face ou de changements profonds et brusques ${ }^{3}$ dans le caractère du journal; et la clause de cession en cas de vente ou de changement d'actionnariat dans l'entreprise.

l'image. La voix over au cinéma, Québec-Paris, Nuit Blanche-Méridiens Klincksieck, 1996, p. 30.

1 M. Simonis, "Bernadette n'était pas pyromane", op. cit., p. 3.

2 En France, celles-ci sont inscrites dans la loi, tandis qu'en Belgique, elles le sont dans les conventions collectives. Cf. S. HOEBEKE \& B. MOUFFE, op. cit., pp. 712 713.

3 La clause de conscience ne peut en effet jouer lorsque les changements d'orientation sont le fait de l'évolution normale d'un journal sur une vaste période de temps, ainsi que le rappelle M. MoLINA, Les journalistes : statut professionnel, libertés et responsabilités, Paris, Victoires, 1989, p. 188. 
Dans la pratique, il semble toutefois que ces principes ne soient que pure précaution théorique en Belgiquel. Quoi qu'il en soit, les conséquences matérielles, et surtout morales de ces deux clauses sont extrêmement importantes, "puisqu'elles consacrent la responsabilité personnelle du journaliste dans l'exercice de sa profession, même s'il est lié à l'entreprise par un contrat de salariat"2.

Par ailleurs, le droit de l'écriture journalistique ${ }^{3}$, dans son volet consacré au "régime de responsabilité", détermine les droits et obligations de la presse qui, en Belgique, consacrent la responsabilité individuelle du journaliste, responsabilité articulée entre un volet pénal et un volet civil.

La responsabilité pénale de la presse a trait aux comportements réputés délictueux commis dans le cadre d'une activité de presse et sanctionnés par des peines d'amende ou d'emprisonnement. Elle concerne les abus de la liberté d'expression, essentiellement le délit de presse, défini comme une infraction de droit commun, comportant la manifestation abusive d'une opinion par le truchement de la presse $^{4}$ : injure, diffamation, fausse nouvelle.

Quant au régime civil, il définit les obligations découlant de fautes pouvant être commises à l'occasion d'un recours à la presse et qui causent un dommage à autrui. Il peut s'ensuivre une mise en cause de l'auteur ou de l'éditeur d'une publication, et le cas échéant la condamnation à des dommages et intérêts, à la condition que le plaignant fournisse la preuve de l'existence d'une faute, d'un préjudice, et d'un lien de causalité entre la faute et le préjudice.

En Belgique, un arrêt du 31 mai 1996 de la Cour de cassation avait déjà révolutionné la jurisprudence concernant les procès de presse. Jusqu'alors, les juges appliquaient généralement la

1 Comme le rappellent B. Grevisse et al., (La presse écrite en Belgique, Bruxelles, Kluwer Editorial, 1998, p. 114), la clause de conscience est inscrite seulement dans la convention paritaire et non dans la loi comme en France.

2 J. DE BRoucker, Pratique de l'information et écritures journalistiques, Paris, C.F.P.J., 1995, p. 16.

3 Qui est ou a été dénommé : droit du journalisme, de la presse, de l'information, de la communication, des médias. Voir E. Derieux, "Le droit de l'écriture journalistique" in J.-L. MARTIN-LAGARDETTE, Le guide de l'écriture journalistique, Paris, La Découverte \& Syros, 2000, pp. 197-198.

4 Nous résumons la définition fournie par Carine Doutrelepont et Daniel Fesler, op. cit., 1996, pp. 191-203. Voir donc ces auteurs pour une approche plus technique, notamment la discussion sur la définition juridique de la presse et du délit de presse, comme sur la responsabilité de la presse. Voir également S. HOEBEKE \& B. MOUFFE op. cit., pp. 600-603. 
responsabilité en cascade au pénal, et la responsabilité cumulative au civil. Mais il demeurait une longue controverse doctrinale et jurisprudentielle $e^{1}$ à laquelle cet arrêt mettait fin "en décidant que la responsabilité en cascade s'applique non seulement en matière répressive mais aussi en matière civile". La cascade repose, rappelons-le, sur l'imputabilité successive de la responsabilité à l'éditeur, puis à l'imprimeur, et au distributeur, lorsque l'auteur d'un article incriminé n'est ni connu, ni domicilié en Belgique.

En restaurant la garantie pour le plaignant de se tourner vers une personne plutôt que vers un groupe, la Cour de cassation rétablissait ${ }^{2}$ la victime dans son droit ${ }^{3}$. Par la même occasion, elle établissait la responsabilité individuelle du journaliste. Corollairement, elle permettait à l'éditeur, à l'imprimeur et au distributeur, de se soustraire à toute poursuite lorsque l'auteur était connu et domicilié en Belgique, consacrant ainsi au civil une impunité dont jouissaient déjà ces derniers au pénal.

En résumé, la cascade considère l'auteur comme le seul titulaire de l'opinion émise, et par conséquent le seul à assumer la responsabilité, car "la loi ne veut qu'une victime"4, semble-t-il.

Rappelons les termes de l'article 25, alinéas 2, de la Constitution, servant à désigner les personnes susceptibles d'être déclarées responsables. Les acteurs de la presse sont divisés en deux catégories :

- D'une part, l'auteur réel : la personne qui a "conçu la pensée et rédigé le texte d'où résulte la faute"s. Il est responsable dans tous les cas.

- D'autre part, les "auxiliaires" 6 l'éditeur, l'imprimeur et le distributeur, qui se bornent à prêter matériellement leur concours à l'auteur. Ils ne sont pas responsables tant qu'ils s'abstiennent de tout contrôle sur le contenu des publications.

1 Voir E. Montero, art. cit., pp. 96-97, notes 4 et 5, qui présente les défenseurs et opposants des deux thèses.

2 On rappelle, en effet, que par l'arrêt de 1996, la Cour de cassation ne faisait que confirmer sa jurisprudence établie depuis un arrêt du 24 janvier 1863. Cf. E. MonTERo, "La responsabilité civile des médias", in A. STROWEL \& F. TULKENS (dir.), Prévention des préjudices causés par les médias, Bruxelles, 1998, p. 98.

3 Car, tant que tous étaient responsables civilement, par le biais de la responsabilité cumulative, personne, en quelque sorte, n'était responsable, et la victime s'en trouvait lésée.

4 D'après S. HoEbeKE et B. MoufFE, op. cit., pp. 439 et 563.

5 Cass. 31 mai 1996, Journal des tribunaux, 28 septembre 1996, p. 597.

6 Terminologie empruntée à E. MONTERO, art. cit., p. 98. 
À côté de cela, le constituant avait prévu une exception : les auxiliaires de la presse peuvent également engager leur responsabilité personnelle "lorsqu'ils prennent une initiative, s'immiscent dans le travail de rédaction, apportent des retouches, commandent ou font écrire l'article de presse litigieux"1. Dans ces cas, ils seront considérés comme coauteurs, pour avoir coopéré directement et principalement à la faute civile.

Mais, comme le souligne J. Polet, cette exception doit être comprise par rapport au lien juridique qui unit le journaliste à l'entreprise de presse et singulièrement avec la loi du 3 juillet 1978 relative au contrat de travail. Dans l'esprit de cette loi, le journaliste est responsable de son dol (une faute intentionnelle, de mauvaise foi, commise avec une intention de nuire), ou de sa faute légère habituelle, ou encore ou de sa faute lourde. L'éditeur n'est quant à lui responsable qu' "en cas de faute légère occasionnelle du travailleur". Résultat : là aussi, très souvent, "l'employeur de presse s'est trouvé exonéré de toute responsabilité"2.

Dejemeppe $^{3}$, Montero ${ }^{4}$, ou encore Jongen ${ }^{5}$, entre autres, ont soutenu que le contexte dans lequel le constituant belge avait fixé le cadre de la responsabilité des journalistes avait évolué de façon significative. Dès lors, il importe de reconsidérer la qualification des acteurs de la presse telle qu'elle fut fixée en son temps par la constitution.

On s'appuiera abondamment sur la dimension énonciative de l'article de presse, pour souligner que, de ce point de vue, l'auteur ne se trouve plus jamais seul à rédiger intellectuellement son article, ni seul à exprimer publiquement le texte d'où résulte éventuellement la faute.

\section{Plaidoyer en faveur de la coréité}

S'il en est ainsi, il paraît quelque peu anachronique de continuer à considérer la cascade en soi, et même son extension au civil, comme

1 Ibid.

2 J. POLET, art. cit., 2001, p. 63.

3 B. Dejemeppe, "La responsabilité pénale", in A. Strowel \& F. Tulkens (dir.), op. cit., pp. 137-145.

4 E. MONTERO, ibid., pp. 95-134.

5 F. JONGEN, "La responsabilité pénale et civile de la presse", Journal des procès, $\mathrm{n}^{\circ} 196,31$ mai 1991, pp. 11-13. 
étant la meilleure des solutions. Certes, un avantage indubitable de la responsabilité en cascade demeure en ce que, en attribuant à l'auteur la responsabilité de ses écrits, elle prévenait la censure ${ }^{\text {privée de la }}$ part des auxiliaires. En revanche, elle pèche en ce qu'elle exonère pénalement et civilement des acteurs importants, attribuant la responsabilité au seul auteur.

D'ailleurs, toutes les voix qui aussi bien dans la profession que chez les scientifiques se sont prononcées pour l'évolution de la responsabilité en cascade fondent l'essentiel de leur argumentation sur le caractère quelque peu suranné de ses dispositions. Là-dessus, Montero écrit :

Le régime de la responsabilité en cascade n'échappe pas à la critique. Il apparaît dépassé (...) et inadapté à l'évolution des pratiques de la presse. S'il se justifie à l'égard de l'imprimeur et du distributeur, il ne s'impose nullement en ce qui concerne l'éditeur ${ }^{2}$.

Et de préconiser, avec Dejemeppe, et à la suite de Jongen, la remise en question du principe de responsabilité en cascade. D'autres arguments peuvent être invoqués ici. Entre autres :

il n'est pas sans intérêt, dans une démarche qui aboutit à sanctionner le comportement d'un journaliste professionnel, d'examiner de près les règles de pratique que la profession a élaborée ${ }^{3}$.

Or, celles-ci démontrent précisément que l'exception d'antan est devenue la règle, à savoir que dans tous les cas : si l'imprimeur et le distributeur sont bien des "auxiliaires", par contre l'éditeur, de même que d'autres acteurs non identifiés par la loi, c'est-à-dire le rédacteur en chef et le secrétaire de rédaction ne le sont point. Ces derniers ne se contentent pas (ni ne peuvent se contenter) de prêter un concours

1 On attirera l'attention du lecteur sur un usage courant et abusif de ce terme. Car, stricto sensu, l'exercice normal de son autorité hiérarchique par l'éditeur ne constitue guère de la censure. Nous remercions Jacques Polet à qui nous devons la pertinence de cette précision.

2 E. MONTERo, op. cit., p. 99. Et Dejemeppe (ibid. p. 143) de renchérir : "pour la responsabilité en cascade, la préoccupation originaire du constituant d'éviter la censure du journaliste par l'éditeur ne correspond plus toujours aux réalités d'aujourd'hui. En effet, le monde de la presse est tel que l'équilibre s'est inversé...".

3 M. SimONis, "Comme à la récréation", La Revue Nouvelle, sept. 2001, p. 54. 
matériel à l'auteur. Au contraire, dans la pratique ce sont des personnes qui exercent (et doivent exercer) un contrôle et une surveillance sur le contenu des publications. Ce faisant, ils coopèrent directement et principalement à l'article de presse, même s'ils ne l'ont pas rédigé.

Nous partageons l'avis de Hoebeke et Mouffe selon lequel "s'il est de l'intérêt général que ceux qui n'ont pas participé à la rédaction d'un texte puissent échapper en principe aux actions judiciaires, rien n'empêche l'un ou l'autre de ces intervenants d'encourir une part de responsabilité s'il peut être directement associé à l'opinion émise. S'il a coopéré directement et principalement à la rédaction de l'écrit, il sera poursuivi, le cas échéant, comme coauteur"'.

Considérant donc l'importance de leur responsabilité énonciative, trois acteurs devraient à notre entendement se voir obligés d'assumer la responsabilité pénale et civile de la presse aux côtés du journaliste.

1. Premièrement l'éditeur, pour sa position idéologique : parce qu'il peut être l'instigateur ou l'inspirateur du texte ${ }^{2}$; parce qu'il joue un rôle objectif dans le contenu des articles en les cautionnant ou en les orientant ${ }^{3}$. Il convient sans doute de rappeler que tout article n'est jugé publiable que s'il s'inscrit dans les limites imposées par la ligne éditoriale. Est-il dès lors normal que l'on continue de "feindre d'ignorer la part de responsabilité qui incombe à ceux qui définissent la ligne rédactionnelle et la politique commerciale du journal"' ?

Ensuite pour les dividendes économiques que l'éditeur retire des bons articles de ses journalistes. Témoignage d'un acteur de la presse, J.-F. Dumont :

Dans plusieurs médias, l'éditeur intervient déjà, non pas la plume ou le dictaphone en main, mais en réclamant à sa rédaction une adéquation active aux exigences du marché. Et d'une manière générale, l'entreprise de presse, qui a vocation à dégager du profit, trouve son compte dans les audaces journalistiques, commercialement porteuses. Le droit ne peut pas ignorer cette réalité-là ${ }^{5}$, conclut-il.

1 S. HOEBEKE \& B. MOUFFE, op, cit., p. 562.

2 B. DEJEMEPPE, art. cit., p. 143.

3 E. MONTERo, art. cit., p. 99.

4 Ibid., p. 100.

5 J.-F., DumonT, "Intouchable, le journaliste ?", Le Vif/L'Express, $18^{\circ}$ année, $\mathrm{n}^{\circ} 47$, 26 novembre - 2 décembre 1999, p. 5. 
Tout cela revient à affirmer que l'éditeur participe d'une manière ou d'une autre à l'énonciation du discours de la presse, non seulement en tant qu'inspirateur de la ligne éditoriale, mais aussi, et peut-être surtout, en tant que patron. Il nous paraît donc raisonnable que sa responsabilité judiciaire soit engagée en même temps que celle de l'auteur.

Aussi n'avons-nous aucune réticence à suivre R.O. Dalcq qui, dans ses enseignements, a toujours professé qu'un éditeur peut s'avérer responsable ou coresponsable par le simple fait de la publication des informations. Il s'en explique :

L'éditeur a agi avec négligence ou imprudence en permettant la publication de l'article dommageable et sans se soucier si le journaliste avait vérifié ses sources d'information ; peu importe qu'il n'ait pas coopéré à la rédaction même de l'écrit, le fait de la seule publication peut suffire ${ }^{l}$.

De ce point de vue, la position du législateur français, par rapport au belge ${ }^{2}$, nous paraît plus défendable. Pour le premier, en effet, "considéré avant tout comme une œuvre collective le journal appartient à son «éditeur responsable» qui prend seul l'initiative de la publication des articles qu'il contient"3.

En d'autres termes, pour Roger Dalcq comme pour Etienne Montero, Benoît Dejemeppe, François Jongen, Jean-François Dumont, et nous-même, la responsabilité énonciative de l'éditeur suffit à engager sa responsabilité devant les tribunaux.

2. Deuxièmement, le rédacteur en chef. Il nous souvient qu'en janvier 2000, lors d'un colloque consacré à la responsabilité des journalistes ${ }^{4}$, un éditeur se plaignait du fait que la cascade en Belgique n'incluait pas le rédacteur en chef! Un an plus tard, Martine Simonis enfonçait le clou, estimant à juste titre qu'en matière de responsabilité, l'absence des rédacteurs en chef crève les yeux. Pour le rôle important qu'ils revendiquent et jouent en matière de déontologie, écrivait-elle, ceux-ci devraient se retrouver "dans les prétoires

1 Voir S. HOEbeKe \& B. MoufFe, op. cit., p. 555.

2 En droit belge, la responsabilité de l'exploitant du média est d'ordre subsidiaire (Ibid., p. 567).

3 Ibid.

4 Organisé par l'Observatoire du récit médiatique, Département de communication, U.C.L., Louvain-la-Neuve. 
pour répondre civilement de fautes qui leur sont, en réalité, bien davantage imputables qu'aux éditeurs responsables..."1.

Le rédacteur en chef, il est vrai, est considéré comme la "tête agissante" d'un média. Il est responsable de la mise en édition et de la diffusion d'un article; il traduit la "ligne éditoriale" (politique ou philosophie du journal); il autorise l'impression et doit, à ce titre, prendre connaissance des publications ${ }^{2}$. Qui donc, davantage que lui, devrait endosser "les responsabilités liées à la ligne éditoriale, aux choix des illustrations, à la politique de titraille" 3 ? Le rédacteur en chef, cela est évident, couvre le texte de son autorité et de sa responsabilité. Il devrait pouvoir agir de même devant les juges.

Dans la pratique cependant, le journaliste, tout comme les lay$o u t^{4}$, les art directors et les illustrateurs endossent de plus en plus de responsabilités et par conséquent le rédacteur en chef est confronté aux motivations personnelles de ses subalternes qui choisissent euxmêmes les thèmes et les sujets, etc.

3. Troisièmement enfin, le secrétaire de rédaction dont le pouvoir énonciatif est si décisif, entre autres lors de la hiérarchisation des matières signifiantes. La hiérarchisation, révèlent à cet effet Labaki et Marthoz, est souvent la meilleure mesure du "parti pris" d'un médias.

Par-dessus tout, on épinglera le fait que le pouvoir du secrétaire de rédaction se révèle on ne peut plus considérable, dès lors qu'il organise les trois matières sémiologiques de l'énonciation afin qu'elles forment un tout cohérent. Il a en effet, le dernier mot sur les textes, les photos, la légende, les titres, la mise en pages...

De ce fait, sa responsabilité énonciative se révèle prépondérante, car le secrétaire de rédaction

peut contribuer à l'orientation de sa publication. Son influence peut s'exercer, ses opinions personnelles peser -même sans que cela soit flagrant - par la mise en valeur de tel article au détriment de tel autre, par la rédaction ou même simplement la présentation d'un titre, par le choix d'une illustration qui

1 M. Simonis, "Bernadette n'était pas pyromane", op. cit., p. 3.

2 S. HOEBEKE \& B. MOUFFE, op. cit., p. 566.

3 M. Simonis, ibid.

4 Au départ, un "lay-out" désignait une maquette très poussée donnant l'impression de ce que sera l'annonce définitive. Désormais, le terme est utilisé pour la maquette et pour la personne qui la fait.

5 M. LABAKI, J.-P. MARTHOZ, Vive le journalisme !, Bruxelles, Éd. Complexe / Vagabundo, 1994, p. 99. 
flattera ou ridiculisera un personnage, un groupe... par la rédaction orientée d'une légende, etc. ${ }^{1}$.

On peut donc admettre que la responsabilité énonciative du secrétaire de rédaction est très lourde, et à nos yeux aussi importante, sinon plus, que celle de l'auteur' ${ }^{2}$. Est-il normal qu' un acteur disposant de tant de pouvoir n'assume jamais ses actes aux yeux de la loi ?

Certes, poser c'est la question c'est déjà y répondre, mais il n'entre nullement dans notre intention de plaider pour une quelconque restauration de la responsabilité cumulative dont les effets pervers ont déjà été dénoncés et corrigés par la cascade.

En revanche, à l'appui de la thèse d'une responsabilité partagée, nous invoquerons l'existence d'une échelle énonciative ${ }^{3}$ au niveau du discours de la presse. En effet, l'analyse des interactions scriptovisuelles agissant au sein de celui-ci démontre que les rédacteurs ne disposent pas tous du même statut, ni des mêmes pouvoirs énonciatifs. Il existe une hiérarchie dont l'importance va ascendante, d'une part des "énonciateurs individuels" (les rédacteurs) vers "l'énonciateur institutionnel" (le journal), et d'autre part, des "énonciateurs dans l'ombre" vers les rédacteurs responsables, en passant par les rédacteurs simples.

L'échelle énonciative permet de comprendre, entre autres, pourquoi "ce ne sont pas les mêmes qui rapportent l'information et qui (tâche plus noble) disent ce qu'il faut en penser"4.

Par ailleurs, on ne le soulignera jamais assez, les articles de presse sont des messages scripto-visuels, et leur énonciation polyphonique. En d'autres termes, nous plaidons pour que le législateur considère un article de presse, non comme du texte, plus de l'image, plus de la mise en pages, mais comme une unité formée de ces éléments inter reliés.

Rappelons, à ce sujet, que l'énonciation iconique n'est pas prise en compte dans le régime de responsabilité de la presse en Belgique. En l'espèce, il y a même un certain anachronisme dans le chef du législateur. En effet, "selon la Cour de cassation, ne relèvent pas dès

1 L. GUÉRY, op. cit., p. 25.

2 Le secrétaire de rédaction peut, avec des guillemets par exemple, donner un tour de dérision, d'ironie ou de mise en doute à une idée. Placer le titre de "docteur" entre guillemets permet d'insinuer que le médecin en question n'est qu'un prétendu docteur. Voir E. MONTERO, art. cit., p. 118, note 80.

3 Sur l'échelle énonciative, voir E. MURHULA Amisi NASHI, op. cit., pp. 302-304.

4 D. BougnouX, op. cit., 1993, pp. 103-104. 
lors de la notion de délit de presse les images, les paroles proférées à la radio et, d'une manière générale, toute communication par des médias autres qu'écrits"'.

Ainsi, l'illustration de presse bénéficie d'un régime propre, c'està-dire le droit à l'image ${ }^{2}$. Mais, celui-ci, comme l'expliquent Hoebeke et Mouffe, "ne se limite pas à la photographie : il s'applique aussi au dessin, à la peinture et à toutes les nouvelles techniques de fixation d'image (film, vidéo, CD-Rom, Internet...)"3.

Mais, il faut aussi savoir qu' "à la différence du système français, dans la législation belge actuelle, le droit à l'image n'est qu'indirectement protégé sur le plan pénal"4.

Conséquence : quand elle eut à traiter du droit de réponse en matière d'image, la Cour de cassation belge fut confrontée à une aporie. Elle envisagea donc qu'un juge pût considérer

qu'un article paru dans un écrit périodique justifie "un droit de réponse" et qu'il constate que les termes et les images figurant dans cet article constituent un tout indivisible et partant, un seul texte, fût-il illustré ${ }^{5}$.

Or, n'a-t-il pas été démontré que l'article de presse c'est toujours une énonciation dont les éléments scripto, iconiques et graphiques forment un tout indivisible ? On se rend compte, sans doute, à quel point les conditions de la coresponsabilité, telles que recensées par Robert ${ }^{6}$, se trouvent ici réunies.

Pour cette raison, et pour tout ce qui a précédé, nous pensons avoir fourni des éléments suffisants pouvant fonder une certaine nécessité pour la responsabilité juridique de s'inspirer de la responsabilité énonciative?.

I S. HOEBEKE \& B. MOUFFE, op. cit., p. 602.

2 Lire à ce sujet S. HoEBEKE \& B. MOUFF, ibid., pp. 289-317; ou encore M. IsGOUR et $\mathrm{B}$. VINÇOTTE, Le droit à l'image, Paris, Larcier, 1998.

3 S. HOEBEKE \& B. MOUFF, ibid., p. 289.

4 M. IsGour et B. VINÇOTTE, op. cit., p. 133.

5 Ibid., p. 131.

6 P. Robert, "La responsabilité civile du journaliste", Auteurs et Médias, n 1-2, 2000, pp. 18-19.

7 Tout comme les juges recourent à l'expertise de psychologues dans l'explication et l'interprétation du comportement de certains prévenus, le recours aux spécialistes des médias, pour un éclairage contextuel du fonctionnement de la presse n'est pas dénué d'intérêt, nous semble-t-il. 
D'aucuns en Belgique ${ }^{1}$ considèrent qu'il ne faudrait pas toucher à la Constitution, notamment à son article 25 consacrant la cascade. Ils n'en reconnaissent pas moins, se basant sur l'article $1384 \mathrm{du}$ Code civil, que rien ne s'oppose à la poursuite de l'éditeur, de l'imprimeur et du distributeur, même lorsque l'auteur est connu et domicilié en Belgique, à condition que ce dernier soit leur employé.

Nous estimons, quant à nous, que la dynamique jurisprudentielle pouvant entraîner le constituant et le législateur belges dans le mouvement évolutif de la société est à encourager. En conséquence, nous osons défendre, en matière de responsabilité de la presse, la thèse de la corréité : entre l'auteur de l'article et les responsables du journal.

Et si, pour l'une ou l'autre raison propre à l'univers judiciaire, cette position était jugée inapplicable ou prématurée, la cascade devrait à tout le moins subir un réaménagement, ou plutôt deux. Premièrement, elle devrait inclure le rédacteur en chef, et surtout le secrétaire de rédaction. Deuxièmement, en tenant compte de l'échelle énonciative du discours de la presse, la responsabilité devrait être imputée successivement au propriétaire (éditeur), puis à ses "lieutenants" (rédacteur en chef et secrétaire de rédaction), et ensuite seulement au journaliste.

Dans tous les cas, nous ne voyons plus de raisons nécessaires et suffisantes pouvant s'opposer à ce que soit engagée la responsabilité juridique d'acteurs participant d'une façon décisive à l'énonciation de la presse, dès lors que leur responsabilité énonciative a été établie.

1 Entre autres, C. DOUTRELEPONT \& D. FESLER, art. cit., p. 201. 